\title{
A new class of anisotropic charged compact star
}

\begin{abstract}
A new model of charged compact star is reported by solving the Einstein-Maxwell field equations by choosing a suitable form of radial pressure. The model parameters $\rho, p_{r}, p_{1}$ and $E^{2}$ are in closed form and all are well behaved inside the stellar interior. A comparative study of charged and uncharged model is done with the help of graphical analysis.
\end{abstract}

Keywords: general relativity, exact solutions, anisotropy, relativistic compact stars, charged distribution
Volume I Issue 5 - 2017

\author{
Ratanpal BS,' Bhar P2 \\ 'Department of Applied Mathematics, The MS University of \\ Baroda, India \\ ${ }^{2}$ Department of Mathematics, Government General Degree \\ College, India
}

\begin{abstract}
Correspondence: BS Ratanpal, Department of Applied Mathematics, Faculty of Technology \& Engineering, The MS University of Baroda, Vadodara-390 00I, India, Tel +919825185736, Email bharatratanpal@gmail.com
\end{abstract}

Received: July 29, 2017 | Published: November 24, 2017

\section{Introduction}

To find the exact solution of Einstein's field equations is difficult due to its non-linear nature. A large number of exact solutions of Einstein's field equations in literature but not all of them are physically relevant. A comprehensive collection of static, spherically symmetric solutions are found in. ${ }^{1,2} \mathrm{~A}$ large collection of models of stellar objects incorporating charge can be found in literature. ${ }^{3}$ Proposed that a fluid sphere of uniform density with a net surface charge is more stable than without charge. An interesting observation of ${ }^{4}$ is that in the presence of charge, the gravitational collapse of a spherically symmetric distribution of matter to a point singularity may be avoided. Charged anisotropic matter with linear equation of state is discussed by. ${ }^{5,6}$ Found that the solutions of Einstein-Maxwell system of equations are important to study the cosmic censorship hypothesis and the formation of naked singularities. The presence of charge affects the values for redshifts, luminosities, and maximum mass for stars. Charged perfect fluid sphere satisfying a linear equation of state was discussed by. ${ }^{7}$ Regular models with quadratic equation of state were discussed by. ${ }^{8}$ They obtained exact and physically reasonable solution of Einstein-Maxwell system of equations. Their model is well behaved and regular. In particular there is no singularity in the proper charge density. ${ }^{9}$ Considered a self gravitating, charged and anisotropic fluid sphere. To solve Einstein-Maxwell field equation they have assumed both linear and nonlinear equation of state and discussed the result analytically. ${ }^{10}$ Extend the work of ${ }^{5}$ by considering quadratic equation of state for the matter distribution to study the general situation of a compact relativistic body in presence of electromagnetic field and anisotropy.

Ruderman $\mathrm{R}^{11}$ investigated that for highly compact astrophysical objects like X-ray pulsar, Her-X-1, X-ray buster 4U 1820-30, millisecond pulsar SAX J 1804.4-3658, PSR J1614-2230, LMC X-4 etc. having core density beyond the nuclear density $\left(\sim 10^{15} \mathrm{gm} / \mathrm{cm}^{3}\right)$ there can be pressure anisotropy, i.e, the pressure inside these compact objects can be decomposed into two parts radial pressure $p_{\perp}$ and transverse pressure $p$ perpendicular direction to $p_{r} . \Delta=p_{r}-p_{\perp}$ is called the anisotropic factor which measures ${ }^{r}$ the anisotropy. The reason behind these anisotropic nature are the existence of solid core, in presence of type $3 \mathrm{~A}$ super fluid, ${ }^{12}$ phase transition, ${ }^{13}$ pion condensation, ${ }^{14}$ rotation, magnetic field, mixture of two fluid, existence of external field etc. Local anisotropy in self gravitating systems was studied by. ${ }^{15,16}$ Demonstrated that pressure anisotropy affects the physical properties, stability and structure of stellar matter. Relativistic stellar model admitting a quadratic equation of state was proposed by ${ }^{17}$ in finch-skea space-time. ${ }^{18}$ Has generalized earlier work in modified Finch-Skea spacetime by incorporating a dimensionless parameter $\mathrm{n}$. In a very recent work ${ }^{19}$ obtained a new model of an anisotropic super dense star which admits conformal motions in the presence of a quintessence field which is characterized by a parameter $\omega$ with $-1<\omega<-1 / 3$. The model has been developed by choosing ansatz. ${ }^{2,21}$ Have studied the behavior of static spherically symmetric relativistic objects with locally anisotropic matter distribution considering the Tolman VII form for the gravitational potential $g_{r r}$ in curvature coordinates together with the linear relation between the energy density and the radial pressure.

Charged anisotropic star on paraboloidal space-time was studied by. ${ }^{22,23}$ Studied anisotropic star on pseudo-spheroidal space time. Charged anisotropic star on pseudo-spheroidal space time was studied by. ${ }^{24}$ The study of compact stars having Matese and Whitman mass function was carried out by. ${ }^{25}$ Motivated by these earlier works in the present paper we develop a model of compact star by incorporating charge. Our paper is organized as follows: In section 2, interior space time and the Einstein-Maxwell system is discussed. Section 3 deals with solution of field equations. Section 4 contains exterior space time and matching conditions. Physical analysis of the model is discussed in section 5. Section 6 contains conclusion.

\section{Interior spacetime}

We consider the static spherically symmetric spacetime metric as,

$$
d s^{2}=e^{\nu(r)} d t^{2}-e^{\lambda(r)} d r^{2}-r^{2}\left(d \theta^{2}+\sin ^{2} \theta d \phi^{2}\right) .
$$

Where $v$ and $\lambda$ are functions of the radial coordinate ' $r$ ' only.

Einstein-Maxwell Field Equations is given by

$$
R_{i}^{j}-\frac{1}{2} R \delta_{i}^{j}=8 \pi\left(T_{i}^{j}+\pi_{i}^{j}+E_{i}^{j}\right),
$$


Where,

$$
\begin{gathered}
T_{i}^{j}=(\rho+p) u_{i} u^{j}-p \delta_{i}^{j}, \\
\text { And } \pi_{i}^{j}=\sqrt{3} S\left[c_{i} c^{j}-\frac{1}{2}\left(u_{i} u^{j}-\delta_{i}^{j}\right)\right], \\
E_{i}^{j}=\frac{1}{4 \pi}\left(-F_{i k} F^{j k}+\frac{1}{4} F_{m n} F^{m n} \delta_{i}^{j}\right) .
\end{gathered}
$$

Here $\rho$ is proper density, $p$ is fluid pressure, $u$ is unit four velocities, $S$ denotes magnitude of anisotropic tensor and $C^{i}$ is radial vector given by $\left(0,-e^{-\lambda / 2}, 0,0\right) . F_{i}$ Denotes the anti-symmetric electromagnetic field strength tensor defined by

$$
F_{i j}=\frac{\partial A_{j}}{\partial x_{i}}-\frac{\partial A_{i}}{\partial x_{j}},
$$

That satisfies the Maxwell equations

$$
F_{i j, k}+F_{j k, i}+F_{k i, j}=0,
$$

And

$$
\frac{\partial}{\partial x^{k}}\left(F^{i k} \sqrt{-g}\right)=4 \pi \sqrt{-g} J^{i}
$$

Where $g$ denotes the determinant of $g_{i j}, A_{i}=(\phi(r), 0,0,0)$ is four-potential and

$$
J^{i}=\sigma u^{i},
$$

Is the four-current vector where $F_{i j}$ denotes the charge density.

The only non-vanishing components of $F_{i j}$ is $F_{01}=-F_{10}$. Here

$$
F_{01}=-\frac{e^{\frac{v+\lambda}{2}}}{r^{2}} \int_{0}^{r} 4 \pi r^{2} \sigma e^{\lambda / 2} d r,
$$

And the total charge inside a radius $r$ is given by

$$
q(r)=4 \pi \int_{0}^{r} \sigma r^{2} e^{\lambda / 2} d r .
$$

The electric field intensity can be obtained from $E^{2}=-F_{01} F^{01}$ , which subsequently reduces to

$$
E=\frac{q(r)}{r^{2}} .
$$

The field equations given by (2) are now equivalent to the following set of the non-linear ODE's

$$
\begin{aligned}
& \frac{1-e^{-\lambda}}{r^{2}}+\frac{e^{-\lambda} \lambda^{\prime}}{r}=8 \pi \rho+E^{2}, \\
& \frac{e^{-\lambda}-1}{r^{2}}+\frac{e^{-\lambda} v^{\prime}}{r}=8 \pi p_{r}-E^{2}, \\
& e^{-\lambda}\left(\frac{v^{\prime \prime}}{2}+\frac{v^{\prime 2}}{4}-\frac{v^{\prime} \lambda^{\prime}}{4}+\frac{v^{\prime}-\lambda^{\prime}}{2 r}\right)=8 \pi p_{\perp}+E^{2},
\end{aligned}
$$

Where we have taken

$$
\begin{aligned}
& p_{r}=p+\frac{2 S}{\sqrt{3}}, \\
& p_{\perp}=p-\frac{S}{\sqrt{3}} .
\end{aligned}
$$

$$
8 \pi \sqrt{3} S=p_{r}-p_{\perp}
$$

\section{Solution of field equations}

To solve the above set of equations (13)-(15) we take the mass function of the form

$$
m(r)=\frac{b r^{3}}{2\left(1+a r^{2}\right)},
$$

Where ' $a$ ' and ' $b$ ' are two positive constants. The mass function given in (19) is known as Matese \& Whitman ${ }^{26}$ mass function that gives a monotonic decreasing matter density which was used by ${ }^{27}$ to model an anisotropic fluid star, ${ }^{28}$ to develop a model of dark energy star, ${ }^{29}$ to model a class of relativistic stars with a linear equation of state and $\mathrm{d}^{30}$ to model a charged anisotropic matter with linear equation of state.

Using the relationship $e^{-\lambda}=1-\frac{2 m}{r}$ and equation (19) we get,

$$
e^{\lambda}=\frac{1+a r^{2}}{1+(a-b) r^{2}} .
$$

From equation (13) and (20) we obtain

$$
8 \pi \rho=\frac{3 b+a b r^{2}}{\left(1+a r^{2}\right)^{2}}-E^{2} .
$$

We choose $E^{2}$ of the form

$$
E^{2}=\frac{\alpha a r^{2}}{\left(1+a r^{2}\right)^{2}},
$$

Which is regular at the center of the star. Substituting the expression of $E^{2}$ into (21) we get,

$$
8 \pi \rho=\frac{3 b+a(b-\alpha) r^{2}}{\left(1+a r^{2}\right)^{2}} .
$$

To integrate the equation (14) we take radial pressure of the form,

$$
8 \pi p_{r}=\frac{b p_{0}\left(1-a r^{2}\right)}{\left(1+a r^{2}\right)^{2}},
$$

Where $p_{0}$ is a positive constant, the choice of $p_{r}$ is reasonable due to the fact that it is monotonic decreasing function of ' $r$ ' and the radial pressure vanishes at $r=\frac{1}{\sqrt{a}}$ which gives the radius of the star.

From (24) and (14) we get,

$$
v^{\prime}=\frac{\left(b p_{0}+b\right) r-a\left(b p_{0}+\alpha-b\right) r^{3}}{\left(1+a r^{2}\right)\left[1+(a-b) r^{2}\right]} .
$$

Integrating we get,

$$
v=\log \left\{\frac{C\left(1+a r^{2}\right)\left(\frac{2 b p_{0}+\alpha}{2 b}\right)}{\left[(b-a) r^{2}-1\right]\left[\frac{\left(b^{2}-2 a b\right) p_{0}+b^{2}-\alpha a}{2 b^{2}-2 a b}\right]}\right\},
$$

Where $C$ is constant of integration, and the space time metric in the interior is given by 


$$
d s^{2}=\left\{\frac{c\left(1+a r^{2}\right)\left(\frac{2 b p_{0}+\alpha}{2 b}\right)}{\left[(b-a) r^{2}-1\right]\left[\frac{\left(b^{2}-2 a b\right)}{2 b^{2}-2 a b}\right] p^{2}-\alpha a}\right] d t^{2}-\left[\frac{1+a r^{2}}{1+(a-b) r^{2}}\right] d r^{2}-r^{2}\left(d \theta^{2}+\sin ^{2} \theta d \phi^{2}\right) .
$$

From (14), (15) and (18), we have

$$
8 \pi \sqrt{3} S=\frac{r^{2}\left[A_{1}+A_{2} r^{2}+A_{3} r^{4}\right]}{\left[-4+B_{1} r^{2}+B_{2} r^{4}+B_{3} r^{6}+B_{4} r^{8}\right]},
$$

Where, $A_{1}=b^{2} p_{0}^{2}+14 b^{2} p_{0}-12 a b p_{0}+3 b^{2}-12 \alpha a$,

$$
A_{2}=-2 a b^{2} p_{0}^{2}+8 a b^{2} p_{0}-8 a^{2} b p_{0}-2 \alpha a b p_{0}+2 a b^{2}+8 \alpha a b-16 \alpha a^{2}
$$

$A_{3}=a^{2} b^{2} p_{0}^{2}-4 a^{2} b^{2} p_{0}+4 a^{3} b p_{0}+2 \alpha a^{2} b p_{0}-a^{2} b^{2}+4 \alpha a^{2} b-4 \alpha a^{3}+\alpha^{2} a^{2}$

$$
B_{2}=12 a b-24 a^{2}, \quad B_{2}=12 a b-24 a^{2}, \quad B_{3}=12 a^{2} b-16 a^{3} \text { and }
$$
$B_{4}=4 a^{3} b-4 a^{4}$.

From (18) we obtain,

$$
8 \pi p_{\perp}=\frac{\left[4 b p_{0}+C_{1} r^{2}+C_{2} r^{4}+C_{3} r^{6}\right]}{\left[4-B_{1} r^{2}-B_{2} r^{4}-B_{3} r^{6}-B_{4} r^{8}\right]},
$$

Where, $C_{1}=b^{2} p_{0}^{2}-8 a b p_{0}+3 b^{2}-12 \alpha a$,

$$
C_{2}=-2 a b^{2} p_{0}^{2}+8 a b^{2} p_{0}-12 a^{2} b p_{0}-2 \alpha a b p_{0}+2 a b^{2}+8 \alpha a b-16 \alpha a^{2} \text {, }
$$

$$
C_{3}=a^{2} b^{2} p_{0}^{2}+2 \alpha a^{2} b p_{0}-a^{2} b^{2}+4 \alpha a^{2} b-4 \alpha a^{3}+\alpha^{2} a^{2} .
$$

\section{Exterior space time and matching condition}

We match our interior space time (27) to the exterior ReissnerNordström space time at the boundary $r=r$ (where $r$ is the radius of the star.). The exterior space time is given by the line element

$$
d s^{2}=\left(1-\frac{2 M}{r}+\frac{q^{2}}{r^{2}}\right) d t^{2}-\left(1-\frac{2 M}{r}+\frac{q^{2}}{r^{2}}\right)^{-1} d r^{2}-r^{2}\left(d \theta^{2}+\sin ^{2} \theta d \phi^{2}\right) .
$$

By using the continuity of the metric potential $g_{r r}$ and $g_{t t}$ at the boundary $r=r_{b}$ we get,

$$
\begin{aligned}
& e^{v\left(r_{b}\right)}=1-\frac{2 M}{r_{b}}+\frac{q^{2}}{r^{2}}, \\
& e^{\lambda\left(r_{b}\right)}=\left(1-\frac{2 M}{r_{b}}+\frac{q^{2}}{r^{2}}\right)^{-1} .
\end{aligned}
$$

The radial pressure should vanish at the boundary of the star, hence

\begin{tabular}{|c|c|c|c|c|c|c|c|}
\hline Compact star & $M\left(M_{\odot}\right)$ & Mass(km) & Radius(km) & $\mathbf{a}\left(k^{-2}\right)$ & $\mathbf{b}\left(\mathrm{km}^{-2}\right)$ & $\mathbf{u}$ & $z_{s}$ \\
\hline U I820-30 & 1.58 & 2.33050 & 9.1 & 0.012076 & 0.012370 & 0.256099 & 0.431786 \\
\hline PSR J 1903+327 & 1.667 & 2.45882 & 9.438 & 0.011226 & 0.011699 & 0.260524 & 0.444954 \\
\hline U I608-52 & 1.74 & 2.56650 & 9.31 & 0.011537 & 0.012722 & $0.27567 \mid$ & $0.49294 I$ \\
\hline Vela X-I & 1.77 & 2.61075 & 9.56 & 0.010942 & 0.011952 & 0.273091 & 0.484428 \\
\hline PSR J। $16 \mid 4-2230$ & 1.97 & 2.90575 & 9.69 & 0.01065 & 0.012775 & $0.29987 \mid$ & 0.580629 \\
\hline Cen X-3 & 1.49 & 2.19775 & 9.178 & 0.011871 & 0.011371 & 0.239458 & 0.385309 \\
\hline
\end{tabular}
from equation (24) we obtain

$$
a=\frac{1}{r_{b}^{2}} .
$$

Using (33) \& (19) we obtain

$$
b=\frac{4 m}{r_{b}^{3}} .
$$

We compute the values of ' $a$ ' and ' $b$ ' for different compact stars which is given in Table 1 .

Table I The values of 'a' and 'b' obtained from the equation (33) and (34) 


\section{Physical analysis}

To be a physically acceptable model matter density $(\rho)$, radial pressure $\left(p_{r}\right)$, transverse pressure $\left(p_{r}\right)$ all should be non-negative inside the stellar interior. It is clear from equations (22) and (24) it is clear that $p_{r}$ is positive throughout the distribution. The profile of $p_{\perp}$ and $p$ are shown in Figures $1 \& 2$ respectively. From the figure it is clear that all are positive inside the stellar interior.

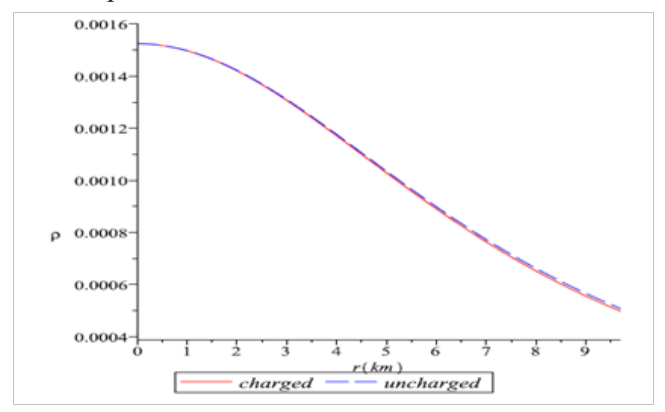

Figure I The matter density is plotted against $r$ for the star PSR JI6|4-2230.

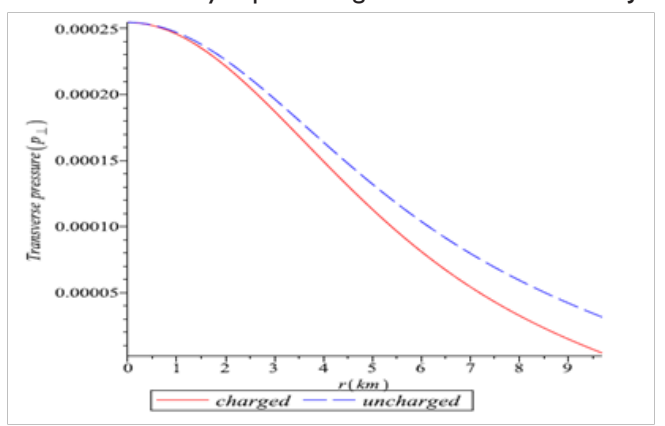

Figure 2 The transverse pressure $p_{t}$ is plotted against $r$ for the star PSR J1614-2230.

The profile of c and $\frac{d p_{\perp}}{d r}$ are shown in Figure 3, it is clearly indicates that $p_{r}, p_{r}$ and $\underset{p_{\perp}}{d r}$ are decreasing in radially outward direction. According to ${ }^{31}$ for an anisotropic fluid spheres the trace of the energy tensor should be positive. To check this condition for our model we plot $\rho-p_{r}-2 p_{\perp}$ against $\mathrm{r}$ in Figure 4. From the figure it is clear that our proposed ${ }^{\perp}$ model of compact star satisfies Bondi's conditions.

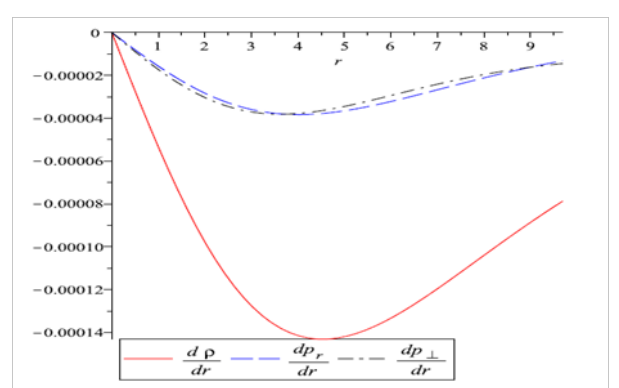
Figure $3 \frac{d \rho}{d r}, \frac{d p_{\perp}}{d r}$ and $\frac{d p_{\perp}}{d r}$ are plotted against $r$ for the star PSR JI6I4-
2230.

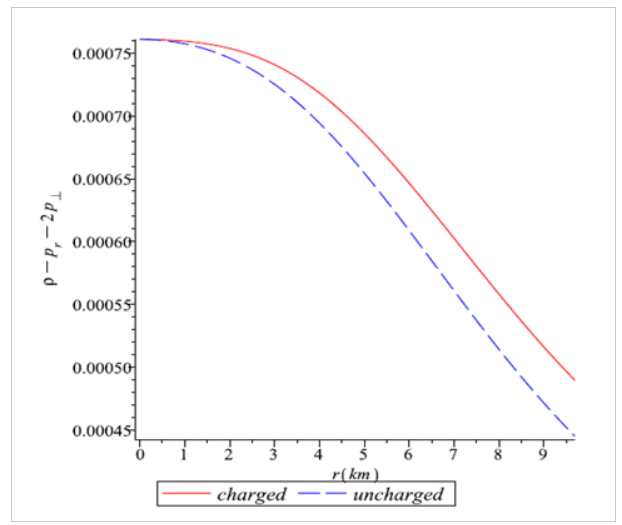

Figure $4 \rho-p_{r}-2 p_{t}$ is plotted against $r$ for the star PSR JI6|4-2230.

For a physically acceptable model of anisotropic fluid sphere the radial and transverse velocity of sound should be less than 1 which is known as causality conditions.

Where the radial velocity $\left(v_{s t}^{2}\right)$ and transverse velocity $\left(v_{s t}^{2}\right)$ of sound can be obtained as

$$
\begin{gathered}
\frac{d p_{r}}{d \rho}=\frac{b p_{0}\left(3-a r^{2}\right)}{5 b+\alpha+a(b-\alpha) r^{2}} . \\
\frac{d p_{\perp}}{d \rho}=\frac{\left(1+a r^{2}\right)^{3}\left[D_{1}+D_{2} r^{2}+D_{3} r^{4}+D_{4} r^{6}+D_{5} r^{8}\right]}{\left.-10 a b-2 a \alpha-2 a^{2}(b-\alpha) r^{2}\right]\left[2+E_{1} r^{2}+E_{2} r^{4}+E_{3} r^{6}+E_{4} r^{8}+E_{5} r^{10}+E_{6} r^{12}\right]} .
\end{gathered}
$$

Where,

$$
\begin{aligned}
& D_{2}=-6 a b^{2} p_{0}^{2}+32 a b^{2} p_{0}-24 a^{2} b p_{0}-4 \alpha a b p_{0}-2 a b^{2}+16 \alpha a b-8 \alpha a^{2}, \\
& D_{2}=-6 a b^{2} p_{0}^{2}+32 a b^{2} p_{0}-24 a^{2} b p_{0}-4 \alpha a b p_{0}-2 a b^{2}+16 \alpha a b-8 \alpha a^{2}, \\
& D_{3}=5 a b^{3} p_{0}^{2}-8 a b^{3} p_{0}+2 \alpha a b^{2} p_{0}-12 a^{2} b^{2} p_{0}+24 a^{3} b p_{0}+6 \alpha a^{2} b p_{0}+7 a b^{3}-12 a^{2} b^{2}-8 \alpha a b^{2}-8 \alpha a^{2} b+24 \alpha a^{3}+3 \alpha^{2} a^{2}, \\
& D_{4}=6 a^{3} b^{b} p_{0}^{2}-6 a^{2} b^{3} p_{0}^{2}+16 a^{2} b^{3} p_{0}-40 a^{3} b^{2} p_{0}-8 \alpha a^{2} b^{2} p_{0}+24 a^{4} b p_{0}+8 \alpha a^{3} b p_{0}+6 a^{2} b^{3}+8 \alpha a^{2} b^{2}-6 a^{3} b^{2}-32 \alpha a^{3} b-2 \alpha^{2} a^{2} b+24 \alpha a^{4}+2 \alpha^{2} a^{3}, \\
& D_{5}=a^{3} b^{3} p_{0}^{2}-a^{4} b^{2} p_{0}^{2}+2 \alpha a^{3} b^{2} p_{0}-2 \alpha a^{4} b p_{0}-a^{3} b^{3}+a^{4} b^{2}+4 \alpha a^{3} b^{2}+\alpha^{2} a^{3} b-8 \alpha a^{4} b+4 \alpha a^{5}-\alpha^{2} a^{4}, \\
& E_{1}=12 a-4 b, E_{2}=2 b^{2}-20 a b+30 a^{2}, E_{4}=12 a^{2} b^{2}-40 a^{3} b+30 a^{4}, E_{4}=12 a^{2} b^{2}-40 a^{3} b+30 a^{4},
\end{aligned}
$$




$$
E_{5}=8 a^{3} b^{2}-20 a^{4} b+12 a^{5} \text { and } E_{6}=2 a^{4} b^{2}-4 a^{5} b+2 a^{6} \text {. }
$$

Due to the complexity of the expression of $\frac{1}{3}<p_{0}<0.3944$ we prove the causality conditions with the help of graphical representation. The graphs of $\left(v_{s r}^{2}\right)$ and $\left(v_{s t}^{2}\right)$ have been plotted in Figures $5 \& 6$ respectively. From the figure it is clear that $0<v_{s t}^{2} \leq 1$ and $0<v_{s t}^{2} \leq 1$ everywhere within the stellar configuration. Moreover $\frac{d p_{t}}{d \rho}$ and $\frac{d p_{r}}{d \rho}$ are monotonic decreasing function of radius ' $\mathrm{r}$ ' for $0 \leq r \leq r$ which implies that the velocity of sound is increasing with the increase of density.

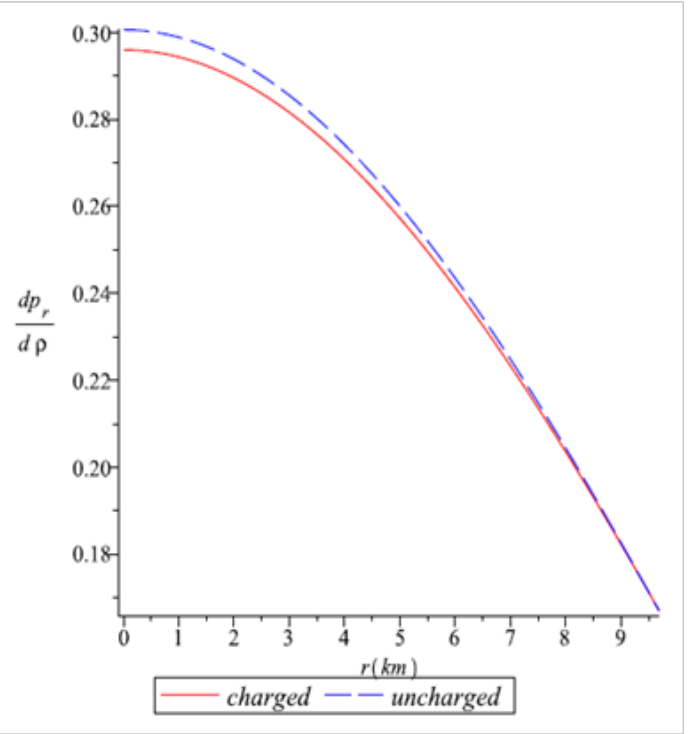

Figure $5 v_{s r}^{2}=\frac{d p_{r}}{d \rho}$ is plotted against $r$ for the star PSR JI614-2230.

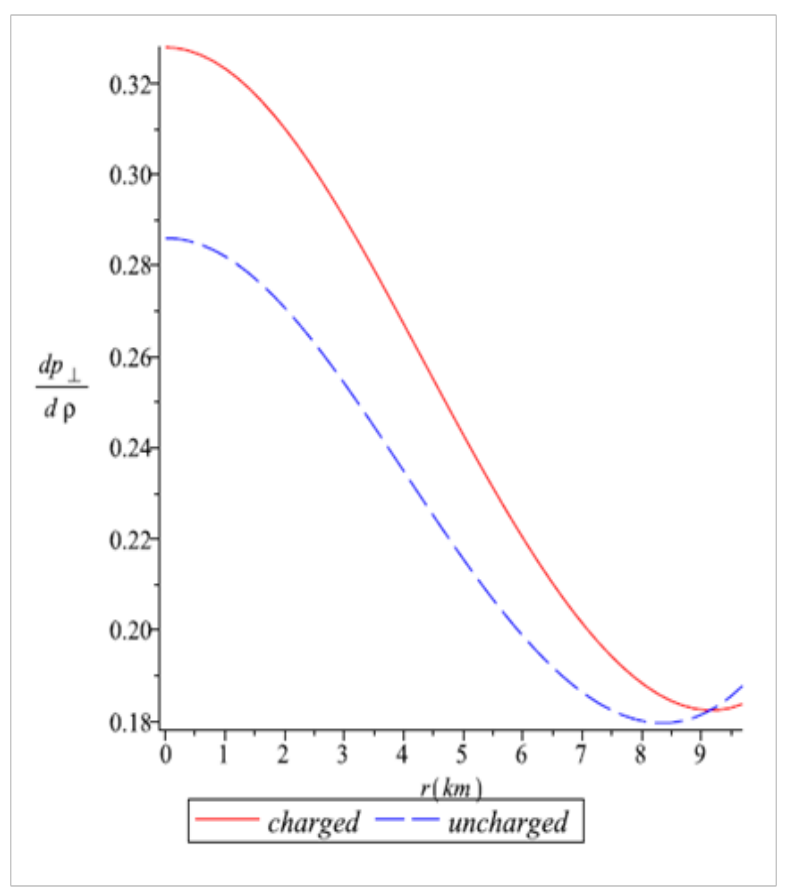

Figure $6 v_{s t}^{2}=\frac{d p_{\perp}}{d \rho}$ is plotted against $r$ for the star PSR J|6| 4-2230.
$\mathrm{A}_{4}$ relativistic star will be stable if the relativistic adiabatic index $\Gamma>\frac{4}{3}$. Where $\Gamma$ is given by

$$
\Gamma=\frac{\rho+p_{r}}{p_{r}} \frac{d p_{r}}{d \rho}
$$

To see the variation of the relativistic index we plot $\Gamma$ for our present of compact star which is plotted in Figure 7. The figure ensures that our model is stable.

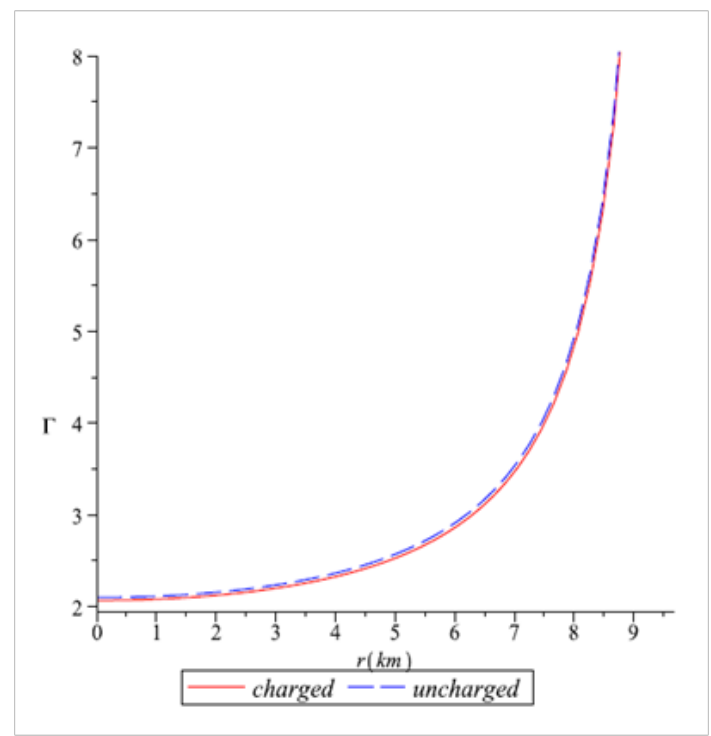

Figure 7 The adiabatic index is plotted against $r$ for the star PSR JI6I 4-2230.

For an anisotropic fluid sphere all the energy conditions namely Weak Energy Condition (WEC), Null Energy Condition (NEC), Strong Energy Condition (SEC) and Dominant Energy Condition (DEC) are satisfied if and only if the following inequalities hold simultaneously in every point inside the fluid sphere.

(i)NEC: $\rho+p_{r} \geq 0$

(ii)WEC: $p_{r}+\rho \geq 0, \rho>0$

(iv) $D E C: \rho>\left|p_{r}\right|, \rho>\left|p_{\perp}\right|$

(iv)DEC: $\rho>\left|p_{r}\right|, \rho>\left|p_{\perp}\right|$

Due to the complexity of the expression of $p$ we will prove the inequality (38)-(41) with the help of graphical representation. The profiles of the L.H.S of the above inequalities are depicted in Figure 8 for the compact star PSR J1614-2230. The figure shows that all the energy conditions are satisfied by our model of compact star (Figures $9 \& 10)$.
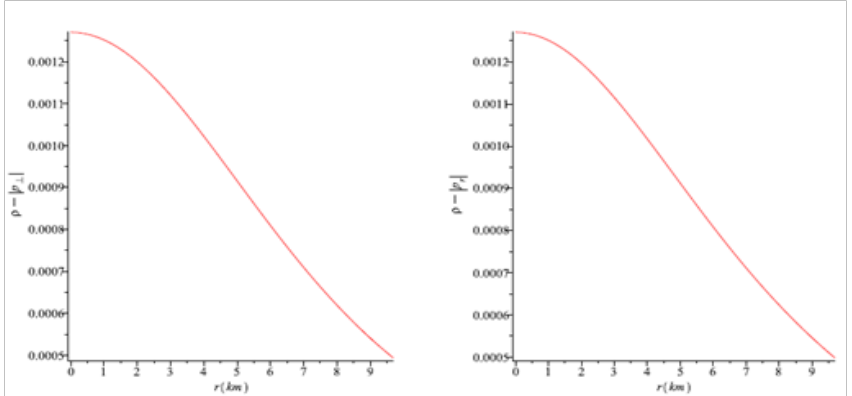


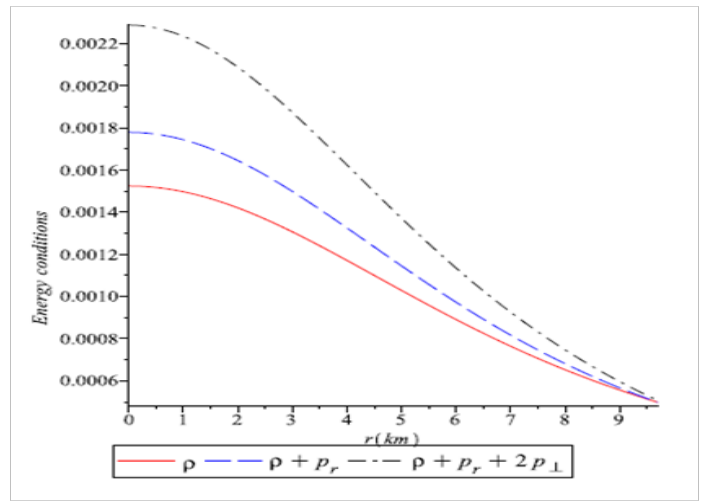

Figure 8 The left and middle figures show the dominant energy conditions where as the right figure shows the weak null and strong energy conditions are satisfied by our model for the star PSR J16|4-2230.

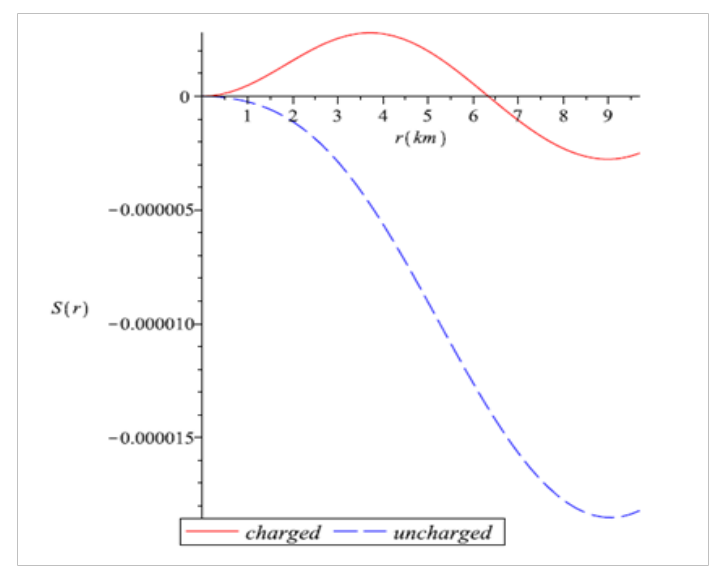

Figure 9 Variation of anisotropy is shown against $r$ for the star PSR JI6I42230.

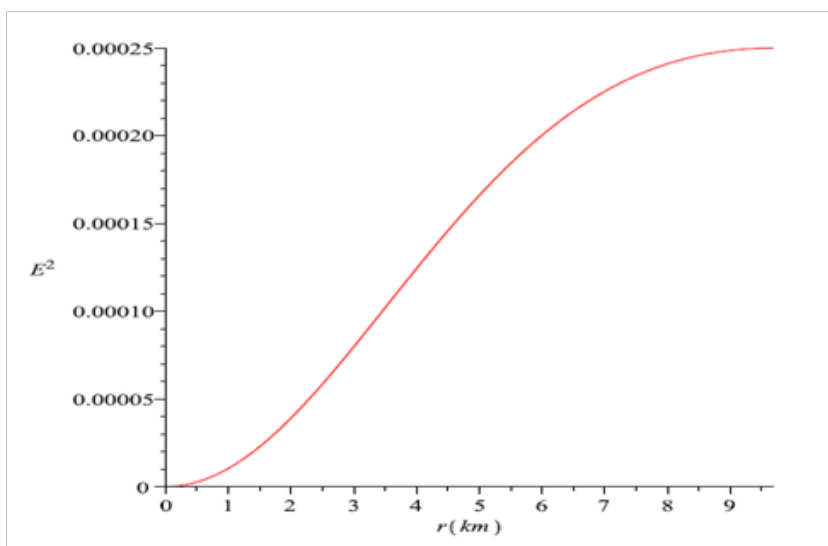

Figure 10 The variation of electric field is shown against $r$ for the star PSR J1614-2230.

The ratio of mass to the radius of a compact star cannot be arbitrarily large. ${ }^{32}$ showed that for a $(3+1)$-dimensional fluid sphere $\frac{2 M}{2}<\frac{8}{9}$. To see the maximum ratio of mass to the radius for our model we calculate the compactness of the star given by

$$
u(r)=\frac{m(r)}{r}=\frac{b r^{2}}{2\left(1+a r^{2}\right)},
$$

and the corresponding surface redshift $z_{s}$ is obtained by,

Therefore $z_{s}$ can be obtained as,

$$
1+z_{s}\left(r_{b}\right)=\left[1-2 u\left(r_{b}\right)\right]^{-1 / 2}
$$

$$
z_{s}\left(r_{b}\right)=\left[\frac{1+(a-b) r_{b}^{2}}{1+a r_{b}^{2}}\right]^{-\frac{1}{2}}-1 .
$$

\begin{tabular}{|c|c|c|c|c|c|}
\hline Compact star & $\begin{array}{l}\text { Central } \\
\text { Density }\left(\rho_{0}\right)\end{array}$ & $\begin{array}{l}\text { Surface } \\
\text { Density }\end{array}$ & $\begin{array}{l}\text { Surface } \\
\text { density }\end{array}$ & $\begin{array}{l}\text { Central } \\
\text { Pressure }\left(p_{0}\right)\end{array}$ & $\left.\frac{d p_{r}}{d \rho}\right|_{r=0}$ \\
\hline & $\mathrm{gm} \cdot \mathrm{cm}^{-3}$ & (uncharged) & (charged) & dyne.cm ${ }^{-2}$ & (charged) \\
\hline U I820-30 & $1.994 \times 10^{15}$ & $6.648 \times 10^{14}$ & $6.514 \times 10^{14}$ & $2.989 \times 10^{35}$ & 0.295227 \\
\hline PSR J I 903+327 & $1.886 \times 10^{15}$ & $6.287 \times 10^{14}$ & $2.827 \times 10^{35}$ & $2.827 \times 10^{35}$ & 0.294958 \\
\hline U I608-52 & $2.051 \times 10^{15}$ & $3.074 \times 10^{35}$ & $3.074 \times 10^{35}$ & $3.074 \times 10^{35}$ & 0.295357 \\
\hline Vela X-I & $1.927 \times 10^{15}$ & $6.423 \times 10^{14}$ & $2.888 \times 10^{35}$ & $2.888 \times 10^{35}$ & 0.295063 \\
\hline PSR JI6|4-2230 & $2.059 \times 10^{15}$ & $6.865 \times 10^{14}$ & $3.087 \times 10^{35}$ & $3.087 \times 10^{35}$ & 0.295376 \\
\hline Cen X-3 & $1.833 \times 10^{15}$ & $6.111 \times 10^{14}$ & $2.748 \times 10^{35}$ & $2.748 \times 10^{35}$ & 0.294815 \\
\hline
\end{tabular}

The surface redshift of different compact stars is given in Table 2.

Table 2 The values of central density, surface density, central pressure and radial velocity of the sound at the origin for different compact stars are obtained 


\section{Conclusion}

We have obtained a new class of solution for charged compact stars having ${ }^{26}$ mass function. The electric field intensity is increasing in radially outward direction and the adiabatic index $\Gamma>\frac{4}{3}$. The physical requirements are checked for the star PSR J1614-2230 and model satisfies all the physical conditions. Some salient features of the model are

In present model if $\alpha=0$, the model corresponds to ${ }^{23}$ model.

In present model if $\alpha=0, a=b=\frac{1}{R^{2}}$, where $R$ is geometric parameter then the model corresponds to ${ }^{R^{2}}$ model, which is stable for $\frac{1}{3}<p_{0}<0.3944$.

\section{Acknowledgments}

BSR is thankful to IUCAA, Pune, for providing the facilities and hospitality where the part of this work was done.

\section{Conflicts of interest}

Authors declare there is no conflict of interest.

\section{References}

1. Stephani H, Kramer D, MacCallum M, et al. Exact Solutions of Einstein's Field Equations, 2nd ed. Cambridge Monographs on Mathematical Physics. Cambridge University Press, New York, USA, 2003. p. 1-25.

2. Delgaty MSR, Lake K. Physical acceptability of isolated, static, spherically symmetric, perfect fluid solutions of Einstein's equations. Computer Physics Communications. 1998;115(2-3):395-415.

3. Stettner R. On the stability of homogeneous, spherically symmetric, charged fluids in relativity. Annals of Physics. 1973;80(1):212-227.

4. Krasinski A. Inhomogeneous cosmological models. 1st ed. Cambridge University Press, USA. 1997.

5. Thirukkanesh S, Maharaj SD. Charged anisotropic matter with a linear equation of state. Classical and Quantum Gravity. 2008;25(23).

6. Joshi PS. Global aspects in gravitation and cosmology. Oxford Science Publications, UK. 1993.

7. Ivanov BV. Static charged perfect fluid spheres in general relativity. Physical Review D. 1992;65(10):104001-1.

8. Takisa MP, Maharaj SD. Compact models with regular charge distributions. Astrophysics and Space Science. 2013;343(2):569-577.

9. Varela V, Rahaman F, Ray S, et al. Charged anisotropic matter with linear or nonlinear equation of state. Physical Review D. 2010;82.

10. Feroze T, Siddiqui AA. Charged anisotropic matter with quadratic equation of state. General Relativity and Gravitation. 2011;43(4):10251035 .

11. Ruderman R. Pulsars: Structure and Dynamics. Annual Review of Astronomy and Astrophysics. 1972;10:427-476.

12. Kippenhahn R, Weigert A. Steller Structure and Evolution. Springer, Berlin, Germany. 1990.
13. Sokolov AI. Phase transitions in a superfluid neutron liquid. Journal of Experimental and Theoretical Physics. 1980;79(4):1137-1138.

14. Sawyer RF. Condensed $\pi$-Phase in Neutron-Star Matter. Physical Review Letters. 1972;29(6).

15. Herrera L, Santos NO. Local anisotropy in self-gravitating systems. Physics Reports. 1972;286(2):53-130.

16. Dev K, Gleiser M. Anisotropic Stars: Exact Solutions. General Relativity and Gravitation. 2002;34(11):1793-1818.

17. Sharma R, Ratanpal BS. Relativistic stellar model admitting a quadratic equation of state. International Journal of Modern Physics D. 2013;22(13).

18. Pandya DM, Thomas VO, Sharma R. Modified Finch and Skea stellar model compatible with observational data. Astrophysics and Space Science. 2015;356(2):285-292.

19. Bhar P. Vaidya-Tikekar type superdense star admitting conformal motion in presence of quintessence field. European Physical Journal C. 2015;75:123

20. Vaidya PC, Tikekar R. Exact relativistic model for a superdense star. Journal of Astrophysics \& Astronomy. 1982;3(3):325-334.

21. Bhar P, Murad MH, Pant N. Relativistic anisotropic stellar models with Tolman VII spacetime. Astrophysics and Space Science. 2015;359:13.

22. Ratanpal BS, Sharma J. Charged anisotropic star on paraboloidal spacetime. Pramana J Phys. 2016;86(3):527-535.

23. Ratanpal BS, Thomas VO, Pandya DM. Anisotropic star on pseudospheroidal spacetime. Astrophysics and Space Science. 2016;361:65.

24. Ratanpal BS, Thomas VO, Pandya DM. A new class of solutions of anisotropic charged distributions on pseudo-spheroidal spacetime. Astrophysics and Space Science. 2015;360:53.

25. Bhar P, Ratanpal BS. A new anisotropic compact star model having Matese \& Whitman mass function. Astrophysics and Space Science. 2016;361:217.

26. Matese JJ, Whitman PG. New method for extracting static equilibrium configurations in general relativity. Physical Review D. 1980;22:1270.

27. Mak MK, Harko T. Anisotropic stars in general relativity. Proceedings of the Royal Society of London A. 2003;459:393-408.

28. Lobo FSN. Stable dark energy stars. Classical and Quantum Gravity. 2003;23(5)

29. Sharma R, Maharaj SD. A class of relativistic stars with a linear equation of state. Monthly Notices of the Royal Astronomical Society. 2007;375(4):1265-1268

30. Thirukkanesh S, Maharaj SD. Charged anisotropic matter with a linear equation of state. Classical and Quantum Gravity. 2008;25(23).

31. Bondi H. The gravitational red shift from static spherical bodies. Monthly Notices of the Royal Astronomical Society. 1990;302(2):337-340.

32. Buchdahl HA. General Relativistic Fluid Spheres. Physical Review journal Achieve. 1959;116(4). 